\title{
Diseño y validación de encuesta sobre motivación de docentes frente a procesos de educación para la sexualidad
}

\author{
Design and validation of a survey on motivation of teachers facing processes of sexuality education
}

\author{
María Constanza Hakspiel-Plata', Lucila Niño-Bautista², Claudia Milena Velazco-Rangel³, Darlen Aragón-Borré4, \\ Liliana Patricia Pineda-Rodríguez ${ }^{5}$
}

1 Enfermera, Esp. Educación y Orientación Sexual. Instituto PROINAPSA - Universidad Industrial de Santander. Bucaramanga, Colombia. e-mail: mconina@yahoo.com

2 Enfermera, MSc en Educación. Instituto PROINAPSA - Universidad Industrial de Santander. Bucaramanga, Colombia. e-mail: lucinino@hotmail.com

3 Enfermera, MSc en Pedagogía (c). Instituto PROINAPSA - Universidad Industrial de Santander. Bucaramanga, Colombia. email: mile0833@hotmail.com

4 Enfermera, MSc en Ciencias de la Familia (c). Instituto PROINAPSA - Universidad Industrial de Santander. Bucaramanga, Colombia. e-mail: aragonborre@gmail.com

5 Psicóloga, Instituto PROINAPSA - Universidad Industrial de Santander. Bucaramanga, Colombia. e-mail: liliana.piedar@gmail.com sobre motivación de docentes frente a procesos de educación para la sexualidad. Univ. Salud. 2016;18(1):46-57. D0I: http://dx.doi.org/10.22267/rus.161801.18

\section{Resumen}

Objetivo: Diseñar y validar encuesta, mediante el modelo de Rasch, para identificar motivaciones internas, externas o desmotivación en docentes de educación básica y media convocados a capacitación en educación para la sexualidad. Materiales y métodos: Estudio de corte transversal cuantitativo con enfoque empírico analítico no experimental para evaluar la encuesta y establecer calidad de los ítems, confiabilidad y validez; la encuesta fue elaborada a partir de la teoría de Deci y Ryan. Participaron 155 docentes de 12 colegios públicos de los municipios de Piedecuesta y El Socorro del departamento de Santander, Colombia. Resultados: Se aplicaron 51 encuestas iniciales y 104 finales. En la prueba inicial solo se logró analizar el componente de motivación externa y se observó un buen ajuste, unidimensionalidad, buena confiabilidad y separación para ítems de 0.97 y 5.76 respectivamente; En la segunda prueba los tres componentes (motivación interna, externa y desmotivación) mostraron ajuste a Rasch con unidimensionalidad, confiabilidad de ítems de 0.80, 0.97 y 0.89 y separación de 2.01, 5.78 y 2.83 respectivamente; no hubo ítems con funcionamiento diferencial por sexo. Conclusiones: Se dispone de encuesta validada y confiable para evaluar motivación docente frente a procesos de capacitación en educación para la sexualidad.

Palabras clave: Estudios de validación; motivación; encuesta; docentes; educación sexual. (Fuente: DeCS, Bireme).

\begin{abstract}
Objective: To design and validate a survey using the Rasch model with the aim of identifying internal and external motivation or discouragement in teachers of basic education and media invited to training in sexuality education. Materials and methods: A quantitative cross-sectional study with non-experimental analytical empirical approach
\end{abstract}


was made in order to evaluate the survey and establish quality items, reliability and validity. The survey was developed from the theory of Deci and Ryan. 155 teachers of 12 public schools from the municipalities of Piedecuesta and El Socorro from the department of Santander, Colombia participated. Results: 51 initial surveys and 104 final ones were applied. In the initial test, it was only possible to analyze the component of external motivation and a good fit, unidimensionality, good reliability and separation for items of 0.97 and 5.76 respectively were observed. In the second test the three components (internal, external and discouragement) showed Rasch fit in terms of unidimensionality, reliability of items of $0.80,0.97$ and 0.89 and separation of $2.01,5.78$ and 2.83 respectively. There were no items with differential performance by sex. Conclusions: There is a validated and reliable survey to evaluate teachers' motivation in front of training processes for sexuality education.

Keywords: Validation studies; motivation; survey; faculty; sex education. (Source: DeCS, Bireme).

\section{Introducción}

En Colombia a partir de 1993 la inclusión de la educación sexual como parte del currículo de las instituciones educativas con programas de preescolar, básica primaria, básica secundaria y media vocacional, tuvo carácter obligatorio mediante la Resolución 3353 del Ministerio de Educación Nacional.1 Posteriormente en 2008, se dio el lanzamiento oficial del Programa de Educación para la Sexualidad y Construcción de Ciudadanía (PESCC) el cual busca generar prácticas pedagógicas que propicien el desarrollo de competencias en niñas, niños, adolescentes y jóvenes, con el fin de que puedan incorporar en su cotidianidad el ejercicio de los derechos humanos sexuales y reproductivos y así tomar decisiones que les permitan vivir una sexualidad sana, plena y responsable.2

La dificultad más importante en este trabajo ha sido desde sus inicios, la resistencia implícita o explícita de buena parte del cuerpo docente, a tener que hacerse responsable de su implementación de acuerdo a lo establecido por la Ley 115 de 1994: "El estudio de estos temas y la formación en tales valores, no exige asignatura especifica. Esta formación debe incorporarse al currículo y desarrollarse a través de todo el plan de estudios. Esto implica la transversalización del proyecto pedagógico en educación para la sexualidad en los planes de estudio y currículos de las instituciones educativas".3

En estudios realizados en Colombia, por el Ministerio de Educación Nacional para evaluar el impacto del Programa PESCC, 4 así como en el desarrollado por Cerón y Cerón en la comunidad educativa de Pasto,5 se identificaron temores en integrantes del colectivo docente al tener que enfrentar ante sus estudiantes los procesos educativos sobre sexualidad, responder inquietudes y aclarar dudas, que ellos mismos aún no han resuelto; lo cual se acompaña de falencias en conocimientos, además que algunos siguen atrapados en patrones religiosos y culturales de prohibición, culpa y machismo que les hace difícil cumplir, con la calidad requerida, para este compromiso de educación en sexualidad.

Esta situación, aún vigente en grupos docentes en Colombia es vivida por docentes de muchos países alrededor del mundo; Bayode ${ }_{6}$ en Nigeria, indagó la percepción de maestros acerca de realizar educación para la sexualidad y encontró que a pesar de la voluntad y el apoyo de las instituciones, el colectivo docente refirió en un $60,9 \%$ que se sentían incómodos enseñando educación sexual y expresaron que algunas de las razones eran no haber recibido ningún tipo de formación, miedo a protestas públicas y a posible acoso legal.

Estos hallazgos son coherentes con lo identificado por Quaresma da Silva en Brasil,,7 sobre la visión de la educación para la sexualidad por parte de docentes de escuelas públicas, así como por Nchia et al.,8 en Camerún sobre los determinantes en las concepciones del profesorado de escuelas primarias y secundarias, alrededor de la educación para la sexualidad; se identificó que los equipos docentes enfrentan como obstáculos para la educación en el tema, el temor a despertar interés sobre éste en el estudiantado, el temor a la censura de la familia y la falta de capacitación, estando principalmente influenciada la educación para la sexualidad, por valores y prácticas tradicionales. 
De igual forma, se encontraron resultados similares en un estudio llevado a cabo en los Estados Unidos por Puchner y Klein,9 en el cual se consultaron experiencias docentes respecto a la educación para la sexualidad; muchos de los maestros entrevistados consideraron beneficioso para el estudiantado saber sobre sexualidad, sin embargo, evitaban el tema expresando que son conservadores, tienen sus propias creencias, sienten molestias por el cuestionamiento de sus opiniones personales, consideraban que las familias podían molestarse si se discutía con las y los estudiantes sobre sexualidad y también tenían conocimiento sobre maestros que fueron sancionados.

Esta situación, vivida por buena parte de los equipos docentes, incide directamente en la motivación que pueden experimentar cuando son convocados a procesos de capacitación para acompañar la educación sexual de sus estudiantes; la motivación puede ir desde la más positiva, pues la oportunidad de recibir capacitación en el área puede ser la solución a sus temores, hasta la ausencia total de motivación, pues por diversas razones, la educación para la sexualidad puede ser un tema sobre el cual no desean hablar.

Indagar sobre los aspectos que pueden motivar a un grupo de docentes de educación básica y media a participar en cursos de actualización o capacitación en un tema controversial como la educación para la sexualidad, es un paso fundamental cuando se quiere avanzar en el mejoramiento de dichos procesos; el interés en la motivación, surgido en el grupo investigador, reside en su potencia explicativa y predictiva de la conducta humana, como lo sustentan Manassero y Vázquez 10 y en su capacidad para poner en movimiento a las personas como lo expresan Legaspi et al.,11 "la motivación es una fuerza de origen psicológico - resultado de la interacción del individuo con una determinada situación- que orienta a las personas hacia la acción".

La construcción teórica para el estudio de los factores o las fuerzas que mueven a una persona a realizar acciones sobre sí mismo, otras personas o su entorno, tiene un largo recorrido y ha pasado por diversos enfoques. Comenzó alrededor de la tercera década del siglo pasado con el enfoque mecanicista en el cual el instinto, la necesidad, el impulso y la eran los desencadenantes de las acciones de los organismos y de allí se pasó al enfoque cognitivista orientado específicamente a la motivación de la conducta humana, con gran énfasis en el estudio de la motivación de logro.10

Otros organizaron el estudio de la motivación en tres grupos dependiendo de la teoría de base que los sustenta: los conductistas explicaron la motivación por la presencia de estímulos externos y refuerzos, lo cual indica que las personas pueden ser movidas a la acción por premios, incentivos o por castigos; los humanistas consideraron que la motivación humana está dada por sus necesidades de libertad, reconocimiento, autoestima, su capacidad de elección y el deseo de autorrealización, y los cognitivistas para quienes la motivación está mediada por la búsqueda del significado, el sentido y la satisfacción por lo que se hace, señalan que la motivación es interna y está guiada por las metas que se tracen y las expectativas que se tengan.12

Investigadores en el tema coinciden en afirmar que hoy no existe una teoría unificada sobre la motivación y por el contrario coexiste una amplia variedad de enfoques dentro de los cuales los fundamentados en perspectivas cognitivas y sociales son los que han desarrollado propuestas nuevas.13,14

Para la realización de la presente investigación se encontró que enfoques cognitivos más recientes han convertido la motivación en eje para el proceso educativo y han identificado la relación existente entre ésta y la curiosidad, el aprendizaje y la perseverancia. Bajo este enfoque se eligió el trabajo desarrollado por Deci y Ryan ${ }_{15}$ quienes han configurado una macro teoría de la autodeterminación -TAD- mediante la cual se estudia hasta qué punto las decisiones que toman las personas son voluntarias y autodeterminadas, además de mediadas por un proceso reflexivo que les lleva a comprometerse con las decisiones tomadas. 
En la toma de decisiones entran en movimiento fuerzas de origen psicológico que hacen interactuar las dos dimensiones que operan en el proceso motivacional: la intrínseca, ligada a los intereses, deseos y expectativas de los individuos y la extrínseca, entendida como los aspectos del contexto que pueden funcionar como estímulos.11 En la interacción de estas dos dimensiones se generan tres tipos de motivación: intrínseca, extrínseca y ausencia de motivación y en cada uno es diferente el grado de autonomía y autodeterminación; las conductas intrínsecamente motivadas son el prototipo de las decisiones autodeterminadas. 16

En el caso de la motivación extrínseca, un meta análisis de 128 investigaciones comprobó que cuando las personas deciden porque hay estímulos monetarios $\mathrm{u}$ otros estímulos contingentes asociados a la conducta, estos socaban la motivación intrínseca y el locus de causalidad pasa de lo interno a lo externo. En la no motivación o desmotivación los sentimientos de autodeterminación no existen y no se dan razones ni extrínsecas o externas ni intrínsecas o internas que sustenten la conducta.17 En la TAD, las necesidades son los mediadores psicológicos que influyen en los 3 tipos de motivación y a su vez en la personalidad y lo afectivo entre otros.18

Vallerand, Blais, Briere, Pelletier en 1989 elaboraron y validaron una encuesta para operacionalizar la TAD; la encuesta, construida inicialmente en francés y posteriormente traducida al inglés, tuvo buenos resultados psicométricos en ambas versiones en concordancia con los planteamientos teóricos de Deci y Ryan.19 Sobre la base de este instrumento se han construido otros más, fundamentados en la TAD por diferentes investigadores los cuales fueron tomados como referentes para la presente investigación como se verá en la metodología.9,11,13

El presente estudio tuvo como objetivo diseñar y validar una encuesta, mediante el modelo de Rasch, fundamentada en la teoría de la autodeterminación de Deci y Ryan, que permitiera identificar las motivaciones internas, externas o la desmotivación en grupos de docentes de educación básica y media convocados a procesos de capacitación para desempeñar mejor su papel de orientadores de la educación para la sexualidad con sus estudiantes.

\section{Materiales y métodos}

Se llevó a cabo un estudio de corte transversal cuantitativo con enfoque empírico analítico no experimental para evaluar encuesta sobre motivación frente a procesos de capacitación en educación para la sexualidad con docentes de educación básica y media de colegios públicos de los municipios vinculados a dos proyectos de intervención; se evaluaron las respuestas dadas por los grupos docentes en la encuesta construida, para establecer la calidad técnica de los ítems y la confiabilidad y validez de la misma utilizando el modelo de Rasch.

Participantes: Para la primera y segunda prueba la muestra fue no probabilística e intencional; en el primer caso, estuvo integrada por 51 docentes de 5 colegios públicos de Piedecuesta que iban a iniciar la capacitación en educación para la sexualidad y que voluntariamente aceptaron participar diligenciando la encuesta del estudio.

En la segunda prueba, también con muestra no probabilística e intencional, respondieron la encuesta 104 docentes perteneciente a siete colegios públicos de El Socorro que habían participado y terminado el proceso de capacitación y que decidieron libremente aceptar la invitación a participar en el estudio.

Instrumento: Para el diseño de la encuesta se partió de la selección y estudio de algunos instrumentos elaborados con base en la teoría de Deci y Ryan; la primera fue la encuesta de motivación en educación - EME- construida en 1989 por Vallerand, Blais, Brière, y Pelletier 19 traducida posteriormente al español y validada por Núñez Martin -Aldo y Navarro;20 ésta encuesta está constituida por 28 ítems sobre motivación intrínseca, extrínseca y desmotivación. Se estudió seguidamente la encuesta de motivación académica-EMAadaptada y validada por Legaspi et al.,11 con 
estudiantes de educación media de una localidad de Buenos Aires - Argentina. Otro escenario educativo en el cual se ha utilizado la EMA es el deportivo en donde Brière, Vallerand, Blais y Pelletier $_{21}$ en 1995 realizaron la construcción y validación del instrumento pertinente.

Una vez analizados estos estudios y sus instrumentos, se tomaron como referentes para la construcción de la encuesta dirigida a docentes de educación básica y media involucrados en procesos de capacitación sobre educación para la sexualidad. Se partió de precisar las definiciones para las tres dimensiones de la motivación y sus siete subdimensiones:

1) Motivación interna: se refiere al hecho de realizar una actividad por sí misma y por el placer y satisfacción derivados de la participación; surge de las necesidades psicológicas innatas de competencia y autodeterminación. Puede ser generada por el interés en: Conocimiento: necesidad de saber, comprender y buscar el significado de las cosas; está relacionada con la exploración, curiosidad y aprendizaje de metas. Ejecución: implicarse en una actividad por el placer y la satisfacción experimentada cuando se intenta lograr o crear algo; superar requerimientos formales superándose a sí mismo. Estímulo: cuando se implica en una actividad para experimentar sensaciones estimulantes como placer sensorial, estético y experiencias emocionales y extraordinarias.

2) Motivación externa: se presenta cuando las conductas emprendidas se realizan para conseguir algún fin u objetivo diferente y no por las conductas en sí mismas. Se divide en: Identificada: las razones externas para la conducta se han internalizado y la persona decide realizarla así no sea gratificante. Introyectada: obedece a razones externas que se han convertido en internas para la persona. Externa: Las conductas están reguladas por recompensas y obligaciones producidas externamente. 3) Desmotivación: se produce cuando las personas perciben una falta de concordancia o interés entre las acciones y los resultados que se obtienen. Hay falta absoluta de motivación intrínseca o extrínseca.
Precisadas las definiciones y tras la lectura y discusión sobre los ítems de los instrumentos de referencia, se procedió a elaborar nuevos, sobre el tema de educación para la sexualidad, para el diseño de una encuesta propia. Los ítems se construyeron teniendo en cuenta la situación de los colectivos docentes ante la reglamentación de ofrecer educación para la sexualidad como parte del currículo; se presentaron como un listado de razones para participar en la capacitación comenzando con la frase: "Participo en procesos de educación para la sexualidad..." A continuación se incluyen ejemplos de los ítems por escala y subescala. Motivación interna -MI- Conocimiento: Por el gusto que tengo cuando aprendo cosas que eran desconocidas para mí. MI -Ejecución: Porque me hace sentir bien darme cuenta que puedo hacer cosas que antes no podía. MI -Estimulación: Por las emociones que me genera el contribuir a la formación más integral de mis estudiantes. Motivación externa -ME- regulación externa: Porque es un lineamiento obligatorio del Ministerio de Educación Nacional. ME- regulación introyectada: Porque la educación sexual beneficia las relaciones en el plano interpersonal. ME - regulación identificada: Porque necesito estar actualizado en los conocimientos respecto al tema. Desmotivación: No obstante, creo que la educación sexual sólo debería llevarse a cabo por profesionales especializados.

La encuesta para la primera prueba estuvo conformada por 52 ítems con el fin de seleccionar los que mostraran los mejores ajustes al final de la misma; como opción de respuesta se utilizó la dicotómica de sí o no. La motivación intrínseca o interna contó con 18 ítems, la motivación extrínseca o externa con 21 ítems y la no motivación o desmotivación con 13 ítems. Una vez se contó con los ítems de la encuesta, se procedió a la revisión y ajuste de léxico, gramática y sintaxis y a mezclar los ítems de las 7 subescalas previo al diligenciamiento.

Procedimiento: Primera prueba: antes de proceder al diligenciamiento de la encuesta se informó a las directivas de las instituciones sobre la investigación y se solicitó y obtuvo su conformidad para invitar al grupo docente; se les dio explicación sobre los objetivos del estudio y la 
importancia de su contribución al logro de los mismos, se informó sobre el carácter voluntario de la participación, la condición de anonimato de su aporte y el manejo responsable y riguroso de la información recopilada. Seguidamente se pidió el consentimiento informado de manera verbal y a quienes no aceptaron participar, se les respetó su decisión; se procedió a entregar la encuesta impresa a quienes permanecieron en el salón y a dar las explicaciones para su diligenciamiento. Se les solicitó subrayar las palabras que fueran confusas o desconocieran su significado o los ítems con dificultad de comprensión. Integrantes del grupo investigador acompañaron el diligenciamiento de la encuesta durante 20 minutos y atendieron preguntas del grupo de docentes.

Segunda prueba: Para la realización de esta prueba se procedió de igual manera que con la anterior en lo relacionado con la información sobre el estudio a directivos y docentes, invitación a participar, respeto hacia la decisión individual de participación y manejo ético de la información. Se solicitó el consentimiento informado en forma verbal y se procedió a entregar la encuesta a quienes permanecieron en el salón; de igual manera, integrantes del grupo investigador estuvieron presentes para atender inquietudes de las y los docentes.

Procesamiento y análisis de datos: Los datos recogidos en cada encuesta se digitaron dos veces en Excel por diferente persona y se hicieron las correcciones de errores detectados quedando las bases de datos listas para transferir al paquete estadístico Stata v $10.0_{22}$ y Winstep $3.8_{23}$ para su análisis, utilizando el modelo de Rasch.24

Los siguientes son los aspectos que se analizaron: i) ajuste de los datos al modelo utilizando el outfit (valores MNSQ, en tablas Winstep) como medida del ajuste; puede ser afectado por los casos atípicos siendo el criterio de aceptación entre el 0.5 y el 1.5. ii) la dimensionalidad de los ítems en la encuesta que informa sobre la pertenencia de éstos a un mismo constructo; se define por los resultados de Infit y Outfit dentro del rango ya mencionado. Esta unidimensionalidad se corrobora por la varianza no explicada en el análisis de componentes principales de los residuales, con un límite de 3.0 autovalores para el primer contraste; iii) la fiabilidad o confiabilidad (Reliability, en tablas Winstep) es la capacidad de reproducir la clasificación de los docentes por medio de la prueba y se consideran aceptables valores de 0.80 o superiores para una prueba confiable. iv) la separación (Separation, en tablas Winstep) indica en qué grado está repartida la dificultad de los ítems a lo largo de la variable en estudio; el índice de separación debería tener un valor mínimo de 2 para considerarse aceptable. v) la distribución de los ítems a lo largo de la escala en mapa de Wright para identificar el nivel de dificultad de éstos y de la encuesta en general y vi) se utilizó la prueba de Mantel-Hanzel como método de estimación del funcionamiento diferencial del ítem -DIF- por sexo. Con los resultados obtenidos en estos indicadores en primera y segunda prueba, se validó el instrumento y su capacidad para evaluar la motivación de docentes de educación básica y media frente a procesos de capacitación para mejorar competencias en educación para la sexualidad.

\section{Aspectos éticos}

Se siguieron las recomendaciones para investigación en seres humanos incluidas en la Declaración de Helsinki 25 y la Resolución 008430 de 1993 del Ministerio de Salud de Colombia;26 que la clasificó como investigación sin riesgo.

\section{Resultados}

En la primera aplicación de la encuesta participaron 51 docentes de los cuales el 71,5\% fueron mujeres con edades comprendidas entre los 30 y 61 años y en la segunda, 104 docentes con la presencia de $71,2 \%$ de mujeres con edades entre los 28 y 63 años.

Primera prueba: Los ítems para motivación interna y desmotivación no ajustaron al modelo de Rasch, en algunos casos presentaron resultados aberrantes con outfit cercano a 6 . Probablemente las condiciones en que se aplicó la encuesta incidieron en estos resultados como se ampliará en la discusión. 
El componente de motivación externa en la encuesta permitió el análisis, sin embargo los ítems 15, 45 y 60 (Porque necesito estar actualizado en los conocimientos respecto al tema; Porque los demás docentes que me rodean creen que es importante y por cumplir con un requisito) no ajustaron mostrando outfit por encima de 3; fueron excluidos del grupo lo cual hizo que el ítem 46 (Porque ahora me sentiría mal si no participo) también quedara por fuera de los límites de aceptación para el outfit, por lo cual se excluyó igualmente del grupo.

De los 16 ítems que quedaron el 50 (Participó en procesos de educación para la sexualidad para no asumir otras actividades laborales) tuvo la máxima medida, pues todas las personas lo respondieron negativamente; los ítems 19, 20, 26 y 27 fueron los más fáciles, pues todos los docentes los respondieron positivamente. Con estas exclusiones, los 11 ítems restantes mostraron: i) ajuste al modelo de Rasch con outfit entre 0,03 y 1,79 ; es de anotar que el outfit por debajo de 0,5 es "menos productivo para la medición, pero no la degrada. Puede provocar de manera engañosa buena fiabilidad y separación" con esta observación, los 6 de 11 ítems con outfit por debajo de 0,5 no se excluyeron, sin embargo quedó el interrogante para la confiabilidad y la separación; ii) hay unidimensionalidad, es decir, estos ítems expresan condiciones de motivación externa; iii) la confiabilidad para los ítems fue de 0,97 y la separación de 5,76 lo cual permite conformar alrededor de 8 grupos; la confiabilidad para las personas fue de 0,70 y la separación de 1,52 ; iv) la medida en lógitos sobre la métrica fue para los ítems de -5,09 a 11,31 y para las personas de $-5,07$ a 10,26 y v) no se encontró funcionamiento diferencial del ítem según el sexo.27

Con los resultados de la primera prueba se eliminaron algunos ítems con problemas de ajuste y se rehicieron muchos otros intentando mejorar la encuesta; se utilizaron frases más precisas, algunas más cortas y se corroboró que estuvieran referidas a un solo aspecto o acción y no a varias. La encuesta quedó conformada por 50 ítems: 18 para motivación interna, 19 para motivación externa y 13 para desmotivación.
Terminado el proceso de análisis, discusión y reelaboración de ítems se hizo la siguiente aplicación de la encuesta con el grupo docente que estaba terminando la capacitación en el municipio de Socorro.

Segunda prueba: Los datos de los 104 docentes que diligenciaron la encuesta mostraron los siguientes resultados luego del análisis mediante el modelo de Rasch:

Motivación interna: Este componente de la encuesta de 18 ítems i) ajustó al modelo con outfit de 0,03 a 1,96 aunque mantuvo ítems por debajo de 0,5 ; ii) mostró unidimensionalidad por las medidas del outfit aunque la varianza inexplicada en el primer contraste estuvo por encima de 3 autovalores, lo cual deja dudas sobre una posible segunda dimensión presente; iii) la confiabilidad de ítems fue de 0,80 y la separación de 2,01 y para las personas la confiabilidad fue de 0,63 con separación de 1,29; iv) la medida en lógitos sobre la métrica para los ítems fue de $-3,06$ a 3,32 y para las personas de $-3,47$ a 4,$24 ;$ v) respecto a la dificultad de los ítems, en éste componente de la encuesta el $56,7 \%$ de las personas respondieron acertadamente los ítems lo cual indica que fueron fáciles para ellas y vi) no hubo funcionamiento diferencial de ítems por sexo.

Motivación externa: De los 17 ítems de este componente se excluyeron $4(15,25,58,59)$ y tres personas que no ajustaron al modelo; los ítems restantes mostraron i) buen ajuste con outfit de 0,05 a 1,57, con 3 ítems por debajo de 0,5; ii) los ítems mostraron ser unidimensionales por medidas de outfit y por la varianza inexplicada en el primer contraste de 2,3 (inferior a 3,0 autovalores); la varianza explicada por los ítems fue del 61\%; iii) la confiabilidad para ítems fue de 0,97 y la separación de 5,78 pudiendo conformar cerca de 9 grupos. La confiabilidad para las personas fue de 0,51 y la separación de 1,02; iv) la medida en lógitos sobre la métrica para los ítems fue de $-4,10$ a 4,18 y para las personas de $-4,01$ a 4,$84 ; \mathrm{v}$ ) respecto a la dificultad de los ítems el 5\% de las personas respondieron positivamente todos los ítems y una persona respondió negativamente todos; vi) no se encontró funcionamiento diferencial de ítems por sexo. 
Desmotivación: Quedaron todos los 13 ítems y personas; i) los ítems mostraron muy buen ajuste con outfit de 0,57 a 1,76 ii) se comprobó unidimensionalidad por medidas de outfit y por varianza inexplicada en primer contraste de 1,8; iii) la confiabilidad para ítems fue de 0,89 y la separación de 2,83 para conformar alrededor de 4 grupos. La confiabilidad para las personas fue de 0,39 y la separación de 0,79 indicando que no se separan, son un solo grupo; iv) la medida en lógitos sobre la métrica para los ítems fue de -1,78 a 2,20 y para las personas de $-2,87$ a 2,$00 ; \mathrm{v}$ ) respecto a la dificultad de los ítems, 3 fueron respondidos correctamente por la totalidad de docentes lo cual indicó que no mostraron ningún grado de dificultad; el $2 \%$ de las personas respondieron positivamente todos los ítems y 15 personas equivalentes al 14,7\% respondieron negativamente todos los ítems; vi) no se encontró funcionamiento diferencial de ítems por sexo. (Tabla 1).

Tabla 1. Resultados de análisis Rasch en segunda prueba de encuesta sobre motivación de docentes frente a procesos de educación para la sexualidad

\begin{tabular}{|c|c|c|}
\hline Estadístico analizado & $\begin{array}{c}\text { Componentes } \\
\text { Encuesta }\end{array}$ & Segunda prueba \\
\hline \multirow{4}{*}{ Ajuste - Outfit ítems (MNSQ) } & Motivación Interna - MI & 0,03 a 1,96 \\
\hline & Motivación Externa - ME & 0,05 a 1,57 \\
\hline & Desmotivación - D & 0,57 a 1,76 \\
\hline & \multirow{3}{*}{ MI } & Unidim. Sí \\
\hline \multirow{7}{*}{$\begin{array}{l}\text { Unidimensionalidad } \\
\text { Varianza explicada } \\
\text { Varianza inexplicada primer } \\
\text { contraste }\end{array}$} & & Var explicada $41,2 \%$ \\
\hline & & Var inex $1^{\mathrm{er}}$ cont 4,0 \\
\hline & \multirow{3}{*}{ ME } & Unidim. Sí \\
\hline & & Var explicada $60,9 \%$ \\
\hline & & Var inex $1^{\mathrm{er}}$ cont 2,3 \\
\hline & \multirow[b]{2}{*}{$\mathrm{D}$} & Unidim. Sí \\
\hline & & Var explicada $27,3 \%$ \\
\hline \multirow{5}{*}{ Confiabilidad } & MI & Personas 0,63 \\
\hline & \multirow{2}{*}{$\mathrm{ME}$} & Ítems 0,97 \\
\hline & & Personas 0,51 \\
\hline & \multirow{2}{*}{$\mathrm{D}$} & Ítems 0,89 \\
\hline & & Personas 0,39 \\
\hline \multirow{5}{*}{ Separación } & MI & Ítems 2,01 \\
\hline & \multirow{2}{*}{$\mathrm{ME}$} & $\begin{array}{l}\text { Personas 1,29 } \\
\text { Ítems 5,78 }\end{array}$ \\
\hline & & Personas 1,02 \\
\hline & \multirow{2}{*}{$\mathrm{D}$} & Ítems 2,83 \\
\hline & & Personas 0,79 \\
\hline \multirow{5}{*}{ Medida de la escala (en lógitos) } & MI & $\begin{array}{l}\text { Ítems }-3,06 \text { a } 3,32 \text { con promedio } 0,0 \\
\text { Personas }-3,47 \text { a } 4,24 \text { con promedio } 3,03\end{array}$ \\
\hline & \multirow{2}{*}{ ME } & Ítems $-4,10$ a 4,18 con promedio 0,0 \\
\hline & & $\begin{array}{l}\text { Personas }-4,01 \text { a } 4,84 \text { con promedio } 0,77 \\
\text { Ítems }-1,78 \text { a } 2,20\end{array}$ \\
\hline & \multirow[t]{2}{*}{$\mathrm{D}$} & con promedio 0,0 \\
\hline & & Personas $-2,87$ a 2,00 con promedio $-1,36$ \\
\hline \multirow{6}{*}{$\begin{array}{l}\text { Personas con puntaje máximo y } \\
\text { mínimo }\end{array}$} & \multirow{2}{*}{ MI } & Max: 59 personas $=56,7 \%$ \\
\hline & & Min: 1 persona $=1 \%$ \\
\hline & \multirow{2}{*}{ ME } & Max: 5 Personas $=5 \%$ \\
\hline & & Min: $=1 \%$ persona \\
\hline & \multirow{2}{*}{ D } & Max: 2 personas $=2 \%$ \\
\hline & & Min: 15 personas $=14,7 \%$ \\
\hline \multirow{3}{*}{$\begin{array}{l}\text { Funcionamiento diferencial de } \\
\text { ítems - FDI }\end{array}$} & Motivación Interna - MI & No \\
\hline & Motivación Externa - ME & No \\
\hline & Desmotivación - D & No \\
\hline
\end{tabular}




\section{Discusión}

La primera acotación se refiere a la estructura de la encuesta siguiendo el diseño de Vallerand, Blais, Brière, y Pelletier ${ }_{19}$ en dimensiones y subdimensiones y el análisis de acuerdo al modelo de Rasch; dado que cada dimensión aborda factores causales diferentes por tipo de motivación (interna, externa y desmotivación) cada una de ellas se comportó como un constructo diferente para el análisis.

Ahora bien, es de resaltar que en los resultados de la primera prueba el único componente o dimensión en que se recogieron respuestas coherentes que permitieron el análisis con Rasch fue en la motivación externa; en motivación interna y desmotivación, las respuestas fueron altamente incoherentes, por tanto no se ajustaron al modelo de Rasch.

Estos resultados se pudieron dar por haber tomado la información de la encuesta en un momento muy inicial cuando el colectivo docente no tenía aun la información suficiente sobre el trabajo que se haría en el curso de capacitación y como ya se ha comentado, hay prevención y resistencia frente el tema de la sexualidad; de hecho una vez se les informó sobre el carácter voluntario de la participación en la encuesta, más de la mitad de los asistentes abandonó el salón.5-7

Sobre los 11 ítems que finalmente quedaron para el análisis de la motivación externa en la primera aplicación, es relevante destacar su confiabilidad de 0.97 y su separación de 5.76; además el hecho que solo una persona contestó todos los ítems de manera correcta, indica que el nivel de dificultad de éstos fue apropiado para docentes que van a iniciar un proceso de capacitación.

Sin embargo, lo más importante como aprendizaje de la primera aplicación de la encuesta fue el estímulo para realizar un ejercicio minucioso y sistemático de revisión y ajuste a los ítems de los tres componentes de la encuesta y que en la segunda aplicación, mostraron mejores resultados como se puede observar en la tabla 1.
Las tres dimensiones de la motivación mostraron ajuste al modelo de Rasch, aunque en motivación interna y motivación externa se tuvieron valores del outfit por debajo de 0,5 ; los valores outfit de la desmotivación fueron los mejores. La unidimensionalidad por valores outfit también se dio en los tres componentes, sin embargo en la motivación interna la varianza inexplicada en el primer contraste por encima de 3 autovalores puede estar indicando una posible segunda dimensión.

En este punto vale la pena referirse a las subdimensiones como orientadoras para la construcción de los ítems las cuales fueron esenciales para que no se quedaran sin incluir elementos que desde la teoría de Deci y Ryan, eran responsables de la motivación respectiva; los efectos favorables se pudieron observar en los buenos resultados de la varianza explicada de la motivación externa, con $60,9 \%$, el valor recomendado en Rasch, y en motivación interna con un valor aceptable de $41,2 \%$. El efecto menos favorable de la construcción de ítems por subdimensión podría ser el responsable de un posible segundo constructo en la motivación interna por tener, alguna de las sub dimensiones, mucho peso en el constructo principal, lo cual es importante observar con detalle en posteriores aplicaciones.

La confiabilidad de los ítems para los tres componentes de la encuesta fue buena, estando, los de la motivación externa y la desmotivación, por encima de 0,80 , valor recomendado por Rasch; estos datos indican que este conjunto de ítems cumplen en principio con los requisitos necesarios para identificar estos tres componentes de la motivación.

La confiabilidad para las personas fue baja debido posiblemente a la homogeneidad del grupo docente, en razón a que todos habían recibido el curso para mejorar sus habilidades en educación para la sexualidad. Dado que la encuesta se hizo pensando en grupos docentes que fueran a iniciar procesos de capacitación en el tema, se requiere incluir nuevos ítems con mayor dificultad para grupos que ya hayan avanzado en la capacitación en el área. 
La mejor separación en los ítems se dio en motivación externa, aunque en los otros dos componentes se podían conformar 3 a 4 estratos. En cuanto a las personas, la separación o no se dio como en la motivación externa y la desmotivación y con esfuerzo se podría llegar a dos estratos o grupos en la motivación interna, confirmando este hecho lo mencionado de la homogeneidad en la motivación del grupo docente.

Respecto de las medidas de ítems y personas en la escala conformada se observó que en la motivación externa y la desmotivación el $47 \%$ de los ítems se situaron por debajo del promedio $(0,0)$ y en la motivación interna el $56 \%$ estuvo sobre el promedio; en todos los componentes hicieron falta ítems de alto nivel de dificultad con énfasis en la desmotivación. El promedio de calificación de las personas en relación con el promedio de los ítems se ubicó 3 lógitos más arriba, mostrando que los ítems de la encuesta fueron muy fáciles para un grupo que acababa de terminar un proceso de capacitación en el tema que les motivó positivamente. En motivación externa las personas se distribuyeron con más normalidad alrededor del promedio y en desmotivación, la mayoría se ubicó por debajo de 0 indicando que encontraron difíciles de responder los ítems.

En los tres componentes de la motivación no se identificaron ítems con funcionamiento diferencial por sexo, lo cual es una fortaleza de la encuesta.

Es de anotar que en los estudios consultados sobre el tema motivación aplicando la teoría de Deci y Ryan para elaborar y validar encuestas, no se encontró ninguno que utilizara el modelo de Rasch en el proceso de validación, tampoco para el análisis de los datos de investigaciones con encuestas validadas. En estudios consultados para el área educativa se utilizaron cuestionarios con escalas tipo Likert y análisis factorial exploratorio o confirmatorio de componentes principales según la teoría clásica de los test, una amplia cantidad de estudios se pueden consultar en el portal de Self-determination theory.28,29
Este estudio de validación de una encuesta sobre motivación dirigida a docentes y utilizando el modelo de Rasch ofrece unos resultados que comparten las fortalezas del mismo, las cuales superan las de la teoría clásica de los test y que se sintetizan en: la invarianza de los parámetros, la estimación del grado de precisión de los ítems y del test, la medición conjunta de ítems y personas, la objetividad específica, las propiedades de intervalo y especificidad del error típico de medida y la capacidad de medir grupos y personalizar la prueba.30-32

Otro punto a comentar es que los estudios sobre motivación en el campo educativo se enfocan en estudiantes; no se encontró, en la literatura consultada, ninguno que explorara la motivación de docentes frente a procesos de capacitación, por tanto la presente investigación parece ser pionera en el área.

\section{Conclusiones}

Se dispone de una encuesta dirigida a docentes de educación básica y media que permite evaluar su motivación frente a procesos de capacitación en educación para la sexualidad, fundamentada en la teoría de la motivación (o de la autodeterminación) de Deci y Ryan y que ha sido validada mediante el modelo de Rasch con resultados adecuados a los parámetros de Rasch. La encuesta puede seguir siendo mejorada en futuras aplicaciones a partir del reconocimiento de que hasta ahora el componente con mejores medidas es el de motivación externa y que los de motivación interna y desmotivación se deben seguir probando y afinando en grupos que aún no hayan pasado por los procesos de capacitación.

Para el grupo investigador se abre una línea de trabajo enfocado a mejorar los procesos de capacitación docente en educación para la sexualidad, comenzando por disponer de un buen instrumento que permita medir su motivación antes del inicio del proceso formativo, y a partir de él, orientar mejor los estímulos y los procesos pedagógicos de tal manera que se les apoye efectivamente en la re estructuración de actitudes y en la construcción de nuevas prácticas para que 
logren obtener mejores resultados en su trabajo educativo con sus estudiantes.

El instrumento validado podría ayudar a desarrollar la investigación alrededor de los procesos de capacitación de docentes de educación básica y media, bastante escasos de la mirada investigativa, hasta donde se logró explorar en el campo de ésta investigación; por ello invitamos a otros grupos a aunar esfuerzos y a que se continúe en esta línea que a mediano y largo plazo, podrá aportar de manera importante en la formación de mejores personas, mejores ciudadanas y ciudadanos y mejor talento humano para el desarrollo del país.

\section{Agradecimientos}

La realización del estudio fue posible gracias al espíritu académico y solidario que mostraron todas las profesoras y profesores que de manera voluntaria respondieron la encuesta. Así mismo agradecemos el apoyo que el profesor titular pensionado de la Universidad Industrial de Santander, Luis Carlos Orozco Vargas, nos brindó en todo el proceso metodológico y de análisis de los resultados obtenidos.

\section{Conflicto de interés}

Las autoras declaran que no tienen conflicto de interés de ningún tipo sobre los resultados presentados.

\section{Referencias}

1. Colombia. Ministerio de Educación Nacional. Resolución 3353 de 1993. Por la cual se establece el desarrollo de programas y proyectos institucionales de Educación Sexual en la Educación Básica del País. 1993.

2. Colombia. Ministerio de Educación. Módulo 1. La dimensión de la sexualidad en la educación de nuestros niños, niñas, adolescentes y jóvenes. En Programa de Educación para la Sexualidad y Construcción de Ciudadanía. Bogotá: 6.

3. Colombia. Congreso de la República. Ley 115 de 1994. Por la cual se expide la Ley General de Educación. 1994.

4. Colombia. Ministerio de Educación Nacional. Evaluación del impacto del programa de educación para la sexualidad y construcción de la ciudadanía. Bogotá 2008:21-22.

5. Cerón C, Cerón E, Urdanivia MI. Conocimientos, actitudes y prácticas en sexualidad humana en la comunidad educativa de Pasto. Revista Universidad y Salud. 2005;1(6):1-26.
6. Bayode IP. Teachers' preparedness for sexuality education in nigerian secondary schools. Revista Electronica KEDI Journal of Educational Policy. 2009; $105 p$.

7. Quaresma da Silva DR. Sex education in the eyes of brazilian public school teachers. Creative Education. 2014;5:1418-1427.

8. Nchia L, Tamesse J, Fonkeng G, Clement P. Determinants of Teachers' Conception of Sex Education in Primary and Secondary Schools in Cameroon. IJSR. 2015;4(2):9961002.

9. Puchner L, Klein NA. Skirting the issue: Teachers' Experiences "Addressing Sexuality in Middle School Language Arts". Revista Electrónica RMLE Online: Research in Middle Level Education. 2012;36(1):1-16.

10. Manassero MA, Vázquez A. Análisis empírico de dos escalas de motivación escolar. Revista electrónica de motivación y emoción. Universidad de las Islas Baleares, España 2013;3(5,6):1-20.

11. Legaspi LP, Aisenson G, Valenzuela V, et al. La motivación y el significado de la escuela para los jóvenes. En Memorias II Congreso internacional de investigación de la Facultad de Psicología de la Universidad Nacional de La Plata. Argentina, Noviembre de 2009.

12. Millán-Hernández MV. Factores de motivación relacionado con el aprendizaje en estudiantes de medicina. Trabajo de grado para obtener el título de Médico Cirujano. Universidad del Oriente, Venezuela, mayo de 2008.

13. Manassero MA, Vázquez A. Validación de una escala de motivación de logro. Psicothema, 1998;10(2):333-351.

14. Álvarez-Ramírez LY. Desarrollos preliminares de la escala de motivación (EM1) para adultos, basada en el modelo motivacional de Mc Clelland. Psychol. Av. Discip. Bogotá, Colombia. 2012;6(1):63-75.

15. Deci EL, Ryan RM. Intrinsic motivation and selfdetermination in human behavior; 1985. (Consultado en septiembre 2013) Disponible en: http://selfdeterminationtheory.org/theory

16. Balaguer I, Castillo I, Duda J. Propiedades psicométricas de la escala de motivación deportiva en deportistas españoles. Revista Mexicana de Psicología. 2007;24(2): 197-207.

17. Deci EL, Koestner R, Ryan RM. A meta-analytic review of experiments examining the effects of extrinsic rewards on intrinsic motivation. Psychol Bull. 1999;125(6):62768; discussion 692-700.

18. Moreno JA, Martínez A. Importancia de la teoría de la autodeterminación en la práctica físico-deportiva: Fundamentos e implicaciones prácticas. Cuadernos de Psicología del Deporte. 2006;6(2):39-54.

19. Vallerand RJ, Blais MR, Brière NM, Pelletier LG. Construction et validation de l'Échelle de Motivation en Éducation (EME). Revue canadienne des sciences du comportement. 1989;(21):323-349.

20. Núñez JL, Martín-Albo J, Navarro JG. Validación de la versión española de la Échelle de Motivation en Éducation. Psicothema, 2005;17(2):344-349.

21. Brière NM, Valerand RJ, Blais MR, Pelletier LG. Développement et validation d'une mesure de 
motivation intrinsèque, extrinsèque et $d^{\prime}$ amotivation en contexte sportif: L'échelle de motivation dans les sports. Int. J. Sport psycho. 1995;(26):465-489.

22. Stata Corp. Stata Statistical Software. College Station, Texas: StataCorp LP. 2010.

23. Linacre JM. WINSTEPS Rasch measurement computer program. Versión 3.81 Chicago: Winsteps. 2013.

24. Orozco LC. Medición en salud; diagnóstico y evaluación de resultados. Colombia. Publicaciones UIS. 2010:4-60.

25. Linacre JM. Help for facets rasch measurement. Software: www.winsteps.com.

26. Núñez JL, Martín-Albo J, Navarro JG, Grijalvo F. Validación de la Escala de Motivación Educativa (EME) en Paraguay. Revista interamericana de psicología. 2006;40(3):391-398.

27. Self-determination theory. An approach to human motivation and personality.

28. Prieto G, Delgado AR. Análisis de un test mediante el modelo de Rasch. Psicothema. 2003;15(1):94-100.

29. Jiménez K, Montero E. Aplicación del modelo de Rasch en el análisis psicométrico de una prueba de diagnóstico en matemáticas. Revista digital matemática, educación e internet. 2013;13(1):1-24.

30. Arias-González V. Sintomatología hiperactiva en niños de 5 a 7 años: calibración de dos instrumentos de evaluación mediante modelos de teoría de respuesta a los ítems. Tesis doctoral, Universidad de Salamanca, 2012.

31. Declaración de Helsinki de la asociación médica mundial. Principios éticos para las investigaciones médicas en seres humanos. 2008.

32. República de Colombia. Ministerio de salud. Por la cual se establecen las normas científicas, técnicas y administrativas para la investigación en salud. 2008. 